\title{
AESTHETICS OF FOOD PORN
}

\author{
UKU TOOMING \\ Hokkaido University \\ uku.tooming@gmail.com
}

SUMMARY: Enticing food photography which stimulates its viewers' cravings, often given a dismissive label "food porn," is one of the most popular contents in contemporary digital media. In this paper, I argue that the label disguises different ways in which a viewer can engage with it. In particular, food porn enables us to engage in cross-modal gustatory imaginings of a specific kind and an image's capacity to afford such imaginings can contribute to its artistic merit.

KEY WORDS: food photography, imagination, art, still lifes, pornography

RESUMEN: La fotografía de comida que resulta atractiva, se antoja a los espectadores y que, con frecuencia, recibe la etiqueta despectiva de "food porn", es uno de los contenidos más populares de los medios digitales contemporáneos. En este artículo, argumento que esta etiqueta oculta diferentes modos en los que un espectador puede enfrentarse a ella. En particular, el food porn nos permite participar en proyectos de imaginación gustativa intermodal de un tipo específico y, la capacidad de las imágenes de provocar dichos proyectos imaginativos puede contribuir al mérito artístico.

PALABRAS CLAVE: fotografía de comida, imaginación, arte, bodegones, pornografía

\section{Introduction}

Pleasures of eating are at the center of a plethora of human activities and passions. An obsession with food seems to be on the rise in the early twenty-first century when professional food criticism has been complemented by foodie culture where sufficient enthusiasm seems to be enough to make one an acceptable participant. With the explosion of digital media, food criticism and presentation have become one of the most popular contents on those platforms.

An especially loved content is stylized food photography which figures prominently on, but is not limited to, websites like Pinterest, Instagram, and Twitter (Rousseau 2014; Kozinets et al. 2016; McDonnell 2016). ${ }^{l}$ The popularity of an image tends to correlate

${ }^{1}$ Aside from social media sites, the same kind of arousing imagery can also often be found in cookbooks and cooking journals. 
with the extent to which it depicts culinary excesses, especially highcalorie foods. Since watching those pictures tends to involve pleasurable sensory arousal, the label "food porn" is often applied to them (FP in short). ${ }^{2}$ As actual pornography arouses sexual desire in a setting where the stimulus isn't the "real thing" but its exaggerated image, so does FP seem to arouse food cravings where the stimulus itself is not the real thing but only its embellished representation.

The label is not limited to still images. Videos of cooking and eating can also merit it, which is complicated by the fact that humans are often depicted together alongside the food. ${ }^{3}$ In the case of a moving image, the sexual analogy can also be accentuated by depicting the cooking process (chopping, kneading, and eating) in ways which stress its similarities with sexual activities. Some people who watch food videos might also watch them because of the person and not because of the food depicted.

There are also interesting complex cases. For instance, mukbang, a type of video that is popular in South Korea, features people eating in front of camera, often eating food in huge quantities (Kim 2018). Since mukbang seems to serve a variety of functions for its viewers - to fend off loneliness, to simulate social eating, to learn how to avoid obesity, to admire the eater, etc.- it is difficult to say if it qualifies as food porn. In the latter case, the focus should be on the food that is depicted and on its arousing aspects. This aspect of the food may be quite irrelevant for viewers' motivation to watch mukbang videos. Also, videos of eating challenges, a popular form of entertainment on the Internet, although depicting food, focus their viewers' attention to the capacities and reactions of the eater and not necessarily on the appeal of the food itself.

In this paper, I limit my attention to images of food without any human accompaniment, such as those that are shared across Instagram and Pinterest. The focus here will be only on the sensory content of FP, i.e., on the food itself. Despite the relative murkiness

2 The term goes back to Rosalind Coward's book Female Desire (1984, p. 103) in the context of feminist critique. However, the term "gastro porn" appeared in New York Review of Books already in 1977 and was used to refer to depictions of food in cookbooks that were supposed to increase excitement about the food depicted while inducing the sense of unattainability (Rousseau 2014, p. 748). In that sense, the term "gastro porn" is close in meaning to "food porn" that we are interested in here.

${ }^{3}$ There may also be verbal food porn, exemplified by food descriptions in some novels, for instance.

${ }^{4}<$ https://www.bbc.com/news/magazine-31130947> [accessed: 13/12/2018]. 
of the concept, we can still say that 'food porn' at its core denotes stylized depictions of food in ways that are supposed to be arousing and exciting for the viewer. I focus on photographs because photography seems to be the primary medium of $\mathrm{FP}$, but what I say can be extended to videos as well.

The main question of the paper is what the pleasurable arousal that people get from watching FP consists in. ${ }^{5}$ This question merits philosophical interest because, on the face of it, there is something puzzling about that kind of arousal. It is puzzling because what people watch is not food, but only a (literally) tasteless representation of it. FP images, after all, don't have any flavor properties. However, people seem to enjoy viewing them because the food depicted looks appetizing and delicious. I take it that for something to look delicious is for it to appear as having a flavor that makes it delicious. To look delicious is therefore to seem to have a gustatory property, and this suggests that people are drawn to FP images because they take gustatory pleasure in what they see in the image. But how is it possible to take this kind of pleasure in something that actually lacks flavor?

Given the puzzle, I am going to try to shed some light on our engagement with FP in order to understand its widely shared appeal. I am going to argue that the psychological structure that undergirds people's pleasurable arousal is actually pretty complex. In particular, I will argue that the characteristic pleasure that they get from FP is enabled by what I call "constructive gustatory imaginings". By imagining flavors in response to seeing the visual properties in the image, people can take gustatory pleasure in watching FP images, and by constructing those imagined flavors, people can enjoy something that they might not even enjoy in real life.

The other aim of the paper is to clarify if FP, insofar as it enables us to engage in constructive gustatory imaginings, merits any aesthetic interest. At first glance, the answer might seem to be negative. After all, isn't the point of watching those images just to stimulate one's hunger? FP seems to function as a supernormal stimulus ${ }^{6}$ which holds viewers captive but does not engage their critical capacities and does not require any discriminating sensibility for the appreciation to be possible. There seems to be an automaticity in the behavior of mindlessly clicking from one FP image to the next. However, in this

\footnotetext{
${ }^{5}$ By assuming that pleasurable arousal is what people often get from FP I am not suggesting that everyone who watches FP has a hedonic purpose in mind.

${ }^{6}$ See Barrett 2010.
} 
paper I will argue that such a dismissal is shortsighted because the gustatory imaginings that $\mathrm{FP}$ affords make it in interesting respects similar to the way in which we relate to artworks. Thus, in order to understand the artistic potential of FP, we need to understand the psychological response that it enables.

The plan of the paper is as follows. In section 2, I will clarify the category of FP by distinguishing it from actual pornography and also from other images that have received the label 'porn'. In the next three sections, I will ask what the pleasurable arousal that FP affords consists in. In section 3 , I will consider a way of making sense of this arousal in terms of desire and argue that it is insufficient to account for the arousal's pleasurable aspects. Then, in section 4, I will argue that we can explain the latter by appealing to gustatory imaginings and will elaborate on this idea in section 5 . In section 6 , I will argue, using the comparison between $\mathrm{FP}$ and still life paintings, that FP images, in virtue of affording pleasurable gustatory imaginings, can be artistic achievements. Finally, in section 7, I will suggest a way in which different FP images can be compared in terms of artistic merit.

\section{The Category of FP}

Why should one take interest in food porn as a distinctive category? On the one hand, it is possible to argue that using the term "porn" in the context of food photography confuses more than clarifies. Food porn is not a form of pornography, strictly speaking. Pornography involves representations that its audience attends to in order to get sexually aroused or gratify themselves, ${ }^{7}$ or that are produced with a purpose to arouse sexually (Uidhir 2009). Since sexual arousal is far from a typical response that viewers have to FP, FP obviously doesn't count as pornography in the strict sense. ${ }^{8}$ Perhaps it is then better to just talk about attractive food photography instead?

On the other hand, there is also "porn" in a generic sense which covers far more types of representations than those that are of food (or sex). On social media, you can find the term "porn" being attached to a wide variety of imagery, insofar as its consumers obsessively watch it. Resulting labels range from \#baseballporn and

\footnotetext{
${ }^{7}$ See Rea 2001.

${ }^{8}$ Another difference between FP and sex porn is that the latter can be criticized from a moral standpoint. After all it involves the depiction of people, arguably in a demeaning and objectifying way. One cannot objectify food, however.
} 
\#carporn to \#catporn and \#yarnporn. But why then should we focus on food porn in particular if the generic label is much more encompassing? ${ }^{9}$

It might thus seem that if we consider pornography in its specific sense, FP doesn't count as such, but in its generic sense, FP doesn't stand out among the countless porn labels. In this section, I will give some reasons to think that it does stand out.

The first reason for picking out $\mathrm{FP}$ as a theoretically interesting category is that it is safe to say that it has a more universal appeal than most of other \#porn labels. Attraction to food imagery is a broader human disposition than attraction to images of yarn or baseball, for instance. It has been suggested that people find looking at food, both real and imagined, naturally rewarding, which suggests that the "visual hunger" for food is an evolutionary adaptation (Spence et al. 2016, p. 54). The "food porn" therefore stands out among other "porn" categories on social media by being more than a mere object of niche interest.

Second, parallels between actual pornography and food porn images run deep. They often merit the same predicates. Like actual pornography, FP can be characterized as obscene and even disgusting, but also as titillating and arousing. For instance, there is something obscene about pictures that depict excessively fatty and sugary foods, such as multiple greasy hamburgers or pizzas, laid on top of one another, just as there is something obscene about the depictions of explicit sex acts. Both actual porn and food porn can induce intense arousal in their viewers which sometimes goes against their better judgment and challenges their self-control. There is also a close association between sexual and gustatory appetite (Korsmeyer 2002, p. 168). Since FP and actual pornography are similar in those respects, FP stands out among other \#porn categories in which case the parallels with actual pornography are superficial at best.

\footnotetext{
${ }^{9}$ In a recent paper, Nguyen and Williams (2020) argue that "porn" in its generic sense denotes all those representations that are used for immediate gratification in such a way that one doesn't have to incur the costs of interacting with what is represented. I think that, as an explanation of the generic meaning, their account is plausible and that "food porn" does denote representations that people enjoy without facing the consequences of actual cooking and eating. However, the aim of this paper is to bring out the distinctiveness of representations that are labeled as "food porn" and the generic meaning does not suffice for this task. Also, the stress on the immediacy of gratification adds a pejorative tinge to the term "food porn", which hides the fact that one's response to FP can be pretty sophisticated, as I will argue below.
} 
Also, the puzzle that FP generates has its a counterpart in the case of actual pornography. As noted in the introduction, the attraction to FP is puzzling because the image itself has no gustatory properties. Food porn and actual pornography are rather similar in this respect because the appeal of the latter is not immediately intelligible either. After all, although the pleasure taken in porn simpliciter seems to be sexual, it is not something that could afford sexual intercourse.

Before moving on, I would like to point out that, despite the similarities, FP is somewhat more challenging phenomenon than actual porn. When we consider paradigmatic pornography, then its viewers watch it for the sake of sexual release. Pornography normally serves as a material for masturbation and the motivation to watch porn is tied up with it: after release, there is also no motivation and no pleasure taken in watching it. It is presumably the purpose of getting release that ultimately matters in the case of pornographic engagement and this at least partially explains people's attraction to porn: the latter functions as a fitting stimulus to a (solipsistic) sexual activity. In the case of watching FP, however, there is no clear analogue of masturbation. There may be cases when a viewer eats something alongside watching FP, but this is presumably not what typically happens, and the viewers don't watch FP for the sake of eating. The pleasurable arousal that one gets from watching FP is more lacking in immediate gustatory rewards than the arousal that one gets from porn simpliciter. Because of this, a call for explanation as to why people are pleasurably aroused is more pressing in the case of FP than in the case of actual porn.

\section{Desire and Arousal}

How to understand the pleasurable arousal that is induced by enticing images of food? One possible answer is that the viewers' arousal is explained by their desire to eat what is depicted. Just as seeing actual delicious food causes in us a desire to eat it, especially when we are hungry, so does seeing images of delicious-looking food cause such a desire, or at least so the suggestion would go. It is true that a desire to eat is often a factor in the appreciation of FP, in that hungry people are more motivated to watch FP than those who are satiated. When one looks at the comment sections regarding FP images a typical way to express one's approval of the image is to exclaim how looking at the image makes one hungry or enhances one's desire to eat. There is also evidence which suggests that hungry people allocate 
more attention to enticing images of food than those who are satiated (Piech, Pastorino and Zald 2010).

However, I do not think a desire to eat fully accounts for the pleasurable aspect of the arousal in question. First, while the arousal that one gets from watching FP is a pleasurable experience, having a desire to eat is pleasurable only if there is a good chance of satisfying it (Sinhababu 2017, p. 28). If there is a sufficiently high subjective probability that one can satisfy the desire, one can take anticipatory pleasure in it. On the other hand, if the probability is low, then having the desire would not be pleasurable. In the case of actually seeing appetizing food, the anticipatory pleasure can explain why we take pleasure at the mere sight of it. The pleasure that we get from viewing FP, however, cannot always be anticipatory, given that watching FP might not involve any promise of eating any foods that are similar to the one depicted. Especially when one enjoys watching images of exotic foods, presented in ways that make them look unattainable, one cannot even expect to eat such foods oneself. This suggests, against experience, that the desire that is involved in watching FP should in those cases be unpleasant, given that there is only a small chance of satisfying it. However, it can be nonetheless pleasurable. It is therefore implausible that the pleasurable arousal that we get from FP is always constituted by a desire to eat.

Second, from my own experience I can say that I can take pleasure in watching FP also when I am satiated. The arousal need not fade away after I have eaten my fill. The putative fact that people under satiety are generally less motivated to watch FP does not preclude them from still taking pleasure in it. If the pleasurable arousal were fully explained by a desire to eat, the pleasure should fade when one does not have the desire anymore. But this does not seem to be the case. The enjoyment that one gets from watching FP is not so tightly dependent on an actual gustatory desire.

Thus, in order to fully understand the arousal caused by FP, appealing to desire is not sufficient. Perhaps we can make progress if we look more closely at the kinds of pleasure that FP can afford. After all, it is the pleasurable aspect of the arousal that the considered explanation had difficulties with. We need an explanation as to why watching FP can be pleasurable even when we are satiated and when the probability of eating something that looks like what see in the image is low. 


\section{Pleasure and Arousal}

Thus far we have only considered one kind of pleasure that can be taken in watching FP, anticipatory pleasure. Since it requires a desire to eat and such a desire is not necessary to enjoy watching FP, anticipatory pleasure cannot be the only kind of pleasure one gets from FP. What other possibilities are there?

Could the pleasure that one gets from viewing FP be non-anticipatory, i.e., could it be a pleasure of fulfilment? The relevant fulfilment cannot consist in eating because eating is (usually) not concurrent with watching FP. And even in cases when it is concurrent, the pleasure of watching FP and the pleasure of actual eating seem to be independent of one another. But perhaps the relevant kind of fulfilment consists in coming to perceive something in the picture? However, since the images of FP are visual objects, what one can strictly speaking perceive are visual surface properties of the image like colors and shapes. But if the pleasure that one gets from watching FP is only pleasure from perceiving visual properties then something is amiss in our characterization of the pleasure that one gets from FP. If someone who watches FP just takes pleasure in the arrangement of colors and shapes on the screen or paper, we can say that she is not watching it as a piece of FP. What is seen in the image, i.e., food with its gustatory properties, should be relevant for the pleasure.

So perhaps it is the food seen in the image that one finds enjoyable? However, both the surface properties of the image and the properties of the food seen in the image are visual properties, which means that the pleasure taken in seeing the food that is depicted has no straightforward connection with gustatory properties of the food that is seen in the image. It is still a visual pleasure that one gets from the properties that one sees. This doesn't sound right. As already noted in the introduction, what makes FP distinct from other images is that it concerns food with its gustatory features, and this distinctiveness should somehow factor into viewers' pleasure. ${ }^{10}$ The puzzle was exactly that the pleasure seems to be gustatory while the image itself has no flavor properties.

Since the viewer cannot strictly speaking taste the food that she sees in the image, how can the experience of the image be even partially gustatory? Does it even make sense? A possibility worth

\footnotetext{
${ }^{10}$ This is not to say that visual properties are not relevant in making the pleasure possible. They are a precondition for being able to perceive and process the image in the first place.
} 
considering is that the gustatory experience consists in imagining tasting the depicted food. According to this suggestion, an agent who watches FP and is pleasurably aroused by it imagines tasting the food that is depicted. The object of pleasure would then be the imagined gustatory properties of the food that is seen in the image.

This idea seems to cohere well with empirical data on how our brains engage in simulating of what it would be like to eat the food that is represented by images (Spence 2017). Watching food pictures involves simulating the sensory experience and motor responses that are implicated in food consumption (Elder and Krishna 2012). It also activates the same sensory regions in the brain that are activated when a person actually consumes food. For instance, seeing images of chocolate chip cookies activates taste cortices (Simmons, Martin and Barsalou 2005). Imagining the act of consumption is multisensory and draws together different sensory modalities (Petit et al. 2016). Since flavor perception is multisensory, in that it involves both taste and olfaction and often incorporates information from other modalities as well (Small and Prescott 2005; Prescott 2015; Velasco et al. 2018), it is not just the taste of food that is simulated but its flavor. Imaginings of food consumption can thus simulate multisensory flavor experiences. ${ }^{11}$ The proposal would then be that the pleasure that we take in watching FP can be constituted by imagining the flavor of the food that is depicted (call this 'gustatory imagination') and liking it. ${ }^{12}$ One can engage in enjoyable gustatory imaginings also under satiety and with an awareness that the food depicted is out of reach. Since it is indisputable that we can take pleasure in imagined scenarios and situations, the present proposal is also quite intuitive.

One might be doubtful about the claim that we imagine tasting a food item when we watch a picture of it. Introspectively, we do not seem to actively imagine anything when we take pleasure in clicking from one FP image to the next. I do not think that imaginings have to be conscious, however. Nothing prohibits us from calling the

${ }^{11}$ That watching food representations involves multi-modal imagery has also been suggested by Bence Nanay: <https://www.psychologytoday.com/us/blog/psychologytomorrow/201904/the-cooking-show-paradox $>$ [accessed: 08/4/2020].

${ }^{12}$ A reviewer suggested that the experience might also involve imagined eating. I definitely do not exclude this possibility, especially given that it is consistent with the data that also motor imagery is involved in watching FP. However, one does not need to assume this to argue that watching FP causes gustatory imaginings, which is a more minimal claim, and more secure because of that. In addition, since imagined chewing, biting and swallowing are not as hedonically tinged as imagined flavors, the latter bear more immediately on understanding the pleasure of watching FP. 
sub-personal simulations of tasting that are not necessarily guided by our conscious intentions "imagining". ${ }^{13}$ If one has a strong pretheoretic intuition that they don't merit this label, one may feel free to use an alternative. One possibility would be to talk simply about "cross-modal associations" between seen visual features and gustatory properties that one does not see but still takes pleasure in. Our minds create cross-modal associations between visual features of the image and gustatory properties. ${ }^{14}$ Since the latter are not perceptually present to us when we look at the image, the ability that creates the connection with them contrasts with a purely perceptual capacity. ${ }^{15}$

One could also argue against the present proposal by suggesting that the viewer who perceives a FP image, instead of imagining, perceives that the food that they see in the image is delicious and takes pleasure in that visual percept. Admittedly, I have been assuming a view of visual perception, according to which the contents of vision are sparse and don't include the property of deliciousness. That being said, even if we allow that deliciousness can be visually perceived, the pleasure one takes from watching FP is more specific: it concerns a more determinate gustatory property in virtue of which the food seen is delicious. And, in order to attribute that more determinate property to the food, one has to resort to cross-modal associations between vision and flavor, these associations being imaginative in the broad sense that I've described.

Is there any constitutive role left for gustatory desires on this picture of pleasurable arousal? If the pleasure taken in watching FP is a pleasure of mere imagination, does it mean that the viewer need not have any desire for the depicted food? A negative answer to this question may seem unpalatable to many, especially if one assents to

${ }^{13}$ Those simulations can still be brought under conscious control, at least to an extent.

${ }^{14}$ For evidence of visual-gustatory cross-modal associations which suggest that visual properties of an item track its flavor, see Piqueras-Fiszman and Spence 2012; Spence 2015; Spence et al. 2010. For instance, people tend to associate red with sweetness, blue with saltiness and green with sourness (Spence and Piqueras-Fiszman 2014, p. 118).

${ }^{15}$ Another way to put the present proposal would be to say that in imagining the flavor of what we see we superimpose a gustatory mental image on the visually perceived physical image. This would be an extension of Robert Briscoe's (2018) notion of "make-perceive". Briscoe himself has noted that there are cases of crossmodal make-perceive where imagery that is projected is of a different modality than the percept on which it is projected. His examples include the visual experience of flowers and cheese eliciting olfactory imagery and seeing lips moving causing auditory imagery (2018, p. 163). 
the desire-satisfaction view of pleasure (e.g. Heathwood 2007). How can one take pleasure in something without having a positive conative attitude toward it? Also, arousal itself seems to be a desirous state at its core, indicated by the fact that saying "I am aroused by it but have no desires toward it" seems to make sense only if one also harbors some strong countervailing aversion against the object or is simply deceiving oneself.

I do not want to commit myself to the desire-satisfaction view of pleasure and I am willing to grant that the viewer need not have any desires for the depicted food. However, someone who does not want to go in that direction could argue that the viewer of FP who takes pleasure in imagining tasting the depicted food has an imaginative counterpart of desire towards it. That there are such desire-like states, or i-desires, which do not amount to desires proper, has been argued for by numerous authors (e.g., Doggett and Egan 2012; Currie 2010). I-desires have been postulated to explain our reactions to fictional events and characters that cannot be easily explained by actual desires. If I react to a piece of fiction as if I wanted a villainous character to escape punishment, for instance, it has been suggested that I do not actually want it but have an i-desire for that character to succeed. Although I am skeptical about this idea myself, for the purposes of this paper I want to accommodate this possibility. One could then say that a viewer who takes pleasure in imagining tasting the depicted food also has an i-desire to eat it, even in a situation wherein one lacks any actual desire to eat.

We now have view according to which the pleasurable arousal that one gets from FP is constituted by the pleasure of imagining tasting the depicted food, possibly together with an i-desire to eat it. This proposal solves the puzzle of absent flavor by showing that we can take gustatory pleasure in visual objects: there is still a gustatory aspect to the experience in that the flavor properties, although not perceived, are imagined. In the next section, I will look more closely at what the relevant kind of imagination amounts to.

\section{Constructive Gustatory Imaginings}

The relevant kind of imagination through which we imagine the flavor of what we see can be understood in different ways. One might take it to mean that the viewer imagines what the photographed item would taste like if one were to actually try it. An attempt to replicate the actual experience is also what an appeal to simulation seems to suggest. However, this suggestion is problematic because the pleasure 
that one takes from imagining consumption would then be vulnerable to the viewer's state of knowledge about the causal source of the image. However, it seems that the pleasures of gustatory imagination that FP induces can be somewhat independent of one's knowledge state about the causal source. Let me explain.

The photographs are often manipulated to make the food more exciting to its viewers. FP imagery is often stylized to the point of becoming unrealistic. An embellished representation of food items does not, or at least need not, reflect their actual taste. As for more extreme examples of image manipulation, photos of "ice cream" can be made by using shaving cream or mashed potatoes instead because ice cream melts too quickly in the studio; and motor oil may be used to make food look shiny. Dish soap is used to create bubbles in soda and a longer lasting foam in beer. Cardboard pieces may be placed between pancakes in a stack in order for it not to sink. ${ }^{16} \mathrm{FP}$, because of the image manipulation, is therefore often misleading about the actual flavor of the image's causal source.

Think now of a person who comes to know that the food that she enjoys watching in an image is actually inedible. Does this knowledge inhibit her from taking pleasure in the image? Although it might affect some viewers, I do not think that one's pleasure is necessarily ruined by it. But if we assume that the relevant pleasure is the pleasure of imagined consumption in the sense of imagining what the depicted item would actually taste like, then the viewer's pleasure should be negatively affected by the knowledge about the process that went into producing the image. I assume that most people don't enjoy imagining drinking motor oil or imagining eating shaving cream. Nevertheless, people can take pleasure in watching the image also when they are aware of the manipulation.

It seems, then, that imaginings of what the depicted food would actually taste like cannot wholly explain the ways in which one can take imagination-based pleasure in watching FP. We should therefore make room for a kind of gustatory imagination that does not involve imagining what the depicted item would actually taste like and that is (largely) independent of one's knowledge about the flavor of the depicted item. ${ }^{17}$ This kind of imagination represents the taste of an imagined gustatory object that is different from what was actually

\footnotetext{
${ }^{16}<$ https://petapixel.com/2016/03/24/photos-show-secret-tricks-food-photography/ https://petapixel.com/2012/08/02/random-things-you-can-use-to-make-food-photosmore-appealing/ $>$ [accessed: 27/11/2018].

${ }^{17} \mathrm{~A}$ reviewer expressed doubt regarding the claim that knowledge of the item doesn't affect the experience. As an example, they suggested that if they became
} 
photographed. If it were identical to the image's causal source, then the subject's gustatory imagining would have to respect the flavor of the causal source. And in that case, the viewer's knowledge about the image manipulation would affect the pleasure that they get from their gustatory imaginings. However, if the gustatory object that one imagines tasting is different from the food item that was actually photographed, this problem need not arise because the viewers are not bound to consider the reality behind the image. Let's call such a form of imagination "Constructive Gustatory Imagination" (CGI in short). It is reasonable to assume that CGI can be pleasurable, insofar as the viewer likes the imagined flavor.

A reviewer suggested that perhaps viewers of FP imagine the taste of what the causal source is supposed to represent, not the taste of the causal source. If that were the case, the knowledge about how the image was produced would still be relevant for one's pleasure, but this would not be ruined by one becoming aware of the actual taste. My response is threefold. First, this suggestion is in line with the idea that knowledge about the actual taste is not relevant, which means that is arguably closer to the conception of CGI than to that of reality-congruent imagining. Second, it is not plausible that viewers' pleasure is bound up with knowing the actual intention behind the image. Becoming aware that the causal source was meant to represent a taste that one dislikes need not ruin one's pleasure, insofar as one constructively imagines a flavor that one likes. Third, this proposal assumes that the causal source is still represented by the viewer. However, when engaging with FP, the causal source need not enter into (i.e., be represented by) the content of pleasurable imaginings at all. Rather, the viewer can enjoy what they see by just imagining what a meal with the given visual appearance could taste like.

In the case of constructive imagination, it is to a considerable extent up to the subject what gustatory properties they attribute to the imagined food item. In other words, the viewer plays a role in constructing the gustatory object that she imagines tasting. That being said, the content of CGI isn't entirely independent of the item that was photographed, otherwise it wouldn't constitute a response to

aware that food on shows like The Great British Bake Off or Ugly Delicious tasted bad, this would significantly damage their pleasure. As a response, I am willing to speculate that the viewers of such shows, which depict other people seeking to understand food and cooking, usually have themselves some epistemic interest in watching them, so that understanding the actual taste of the food becomes relevant for the overall enjoyment. In contrast, I assume that in the case of food photography shared on social media, the epistemic interest is less important. 
the image that one sees. There are constraints: arguably, the subject cannot fluently imagine the object having flavors that diverge too radically from the properties that are paradigmatically associated with the visual form of the depicted food. The visual properties of the image and what it depicts guide and constrain viewers' imaginings. ${ }^{18}$ The imaginative activity is thus still anchored in the visual properties of the image by exploiting cross-modal associations between vision one the one hand, and taste and olfaction on the other.

I also propose that CGI is the typical way in which viewers relate to FP imagery. It is true that FP images need not be manipulated for them to cause pleasurable arousal and that often there is no problem of accurately imagining the taste of the depicted item. However, it is reasonable to think that the viewers are usually not that interested in the reality behind the image. What they primarily care about is whether watching FP is enjoyable. Imaginings that aim at accurately representing the causal source are fragile in their capacity to provide enjoyment because the latter is easily ruined by new information regarding the source of the image. Constructive imagination, on the other hand, lacks such vulnerability and is thereby better suited to satisfy the hedonic purpose. The viewer has more freedom in guiding their imaginings in pleasurable ways. Since CGI can also be employed when the image is not manipulated, I take it that CGI is the default response. ${ }^{19}$

That being said, it is also important to stress that to say that FP images afford pleasurable constructive gustatory imaginings is not to say that other pleasures are precluded. The anticipatory pleasure of a hungry person, considered in the previous section, is a state in which a person who watches food pictures can be in. It could also be the case that some people take purely visual pleasure in looking at the pretty colors and shapes that the depicted food has. And some people might try to imagine what the depicted food would actually taste like and take pleasure in it. That being said, I think that pleasure in CGI is the most pervasive kind of reward that one gets from watching FP. In what follows, I will ask about the artistic potential of FP, insofar as it induces CGI.

${ }^{18}$ As an analogy in the context of painting, see Podro (1998, p. 164) on how the design of a painting constrains the fitting imaginings of the viewer. Podro uses Chardin's Return from Market (1739) as an example.

${ }^{19}$ Similarly, I think that the consumers of actual pornography are not usually interested in accurately capturing the experience of the acts that are represented by the images. It is more than doubtful, for instance, that people who like watching incest porn would actually enjoy incest. 


\section{The Artistic Potential of Food Porn}

As I pointed out in the introduction, "food porn" has its label because images that fall under it induce pleasurable arousal in their viewers. Since there are substantial parallels between arousing food images and pornography, the label "porn" seems fitting in the case of FP. Given that people are sometimes inclined to oppose pornography and art, does the similarity between FP and real porn imply that FP can be dismissed as not fitting to be art? It would imply this only if art and pornography were mutually exclusive categories. This is highly doubtful, however.

For instance, a well-known way of arguing for the exclusionist view has been to claim that pornographic images, unlike artistic ones, aim at their audience's response (arousal) which excludes the attention to the manner of achieving that response. Pornographic images, according to this view, are meant to be treated as transparent, which means that their audience is only occupied with the content of the image and the arousal that it causes, not with its formal and aesthetic properties. In other words, what matters is what is depicted, not how it is depicted (Levinson 2005; see also Uidhir 2009). ${ }^{20}$

Van Brabandt and Prinz argue convincingly, however, that this view simplifies our understanding of pornography. Pornography need not only serve the purpose of arousing the viewer. Like art, at least some pornographic works promote their viewer's attention on how the way in which they depict arouses. Thus, the manner in which the image makes arousal possible can be relevant for the viewers and producers of pornographic images. (Van Brabandt and Prinz 2012, pp. 171-172; see also Maes 2011, p. 53). Similarly, in the case of $\mathrm{FP}$, design features of FP bear on the way in which the viewers' gustatory imaginings unfold. One attends to the way in which the visual properties of the image bring to mind the gustatory properties of the imagined flavor object. The manner of how an image arouses can be relevant for engaging with FP as well.

Therefore, even if FP is deemed pornographic, it can still count as art. In this section, I will provide some reasons for thinking that FP has artistic potential in virtue of inducing CGI. My strategy is to compare $\mathrm{FP}$ with an existing art kind and to show that there is a continuity between them. In particular, by making possible constructive imaginings, an FP image can amount to an artistic achievement.

\footnotetext{
${ }^{20}$ Levinson and Uidhir do not hold exactly the same views of pornography, but for the present purposes, the differences are not that substantial.
} 
It is notable that, when we focus on the content of FP images, then they are similar to still life paintings that depict food. It is true that still lifes primarily aim at making food look beautiful, while FP aims at presenting it as delicious. However, one aim does not exclude another and there can be overlaps in what some still lifes and FP images afford. Take, for instance, Still Life with Apples and Grapes by Monet which depicts bright-colored apples alongside with glistening grapes, or Still Life with Fruit, Bottles, Breads by Goya which gives a yellowish glow to the foodstuff that is presented, or the abundance depicted in Still Life with Peaches, Grapes, Plums and Silver-Gilt Shaker by Liégeois. In these paintings, food is depicted in enticing ways and the reward that the viewer gets can be quite similar to the reward that one gets from watching FP. Such still life paintings that depict food do not merely afford purely visual pleasures and it shouldn't be considered as a failure of a painting if it also affords something like pleasurable CGI. ${ }^{21}$ Instead, it should be considered as an achievement. ${ }^{22}$

Both in the case of (a subset of) still lifes and FP images, a fitting response to the image can include a mental activity through which the viewer is given an opportunity to dwell on the arousal that they get from attending to the perceived visual and imagined gustatory features, without having to actually eat anything. There are also possibilities of surprise for the viewer, for instance, when they discover that they are able to take pleasure in things that they might not enjoy if they actually tasted them, or when the image enables them to synthesize visual and flavor properties in a way that they haven't done before. For instance, the soft yellow glow that unites the foreground items in Goya's Still Life with Fruit, Bottles, Breads makes me imagine the flavors of bread and fruit together and as mutually reinforcing, while those flavors are rather disjunct, perhaps even incompatible, in the experience of actually eating fruit and bread together. In such cases, the author of the image has succeeded in moving the viewer to see and imagine food in a novel and rewarding

${ }^{21}$ Here I am rejecting a view according to which a capacity to induce gustatory arousal cannot contribute to the merit of an artwork. This view was defended, for instance, by Schopenhauer (1969 [1819], p. 207f; cited in Korsmeyer 2002, p. 160).

${ }^{22}$ Admittedly, there are still life paintings which arguably lend themselves only to purely visual pleasures. Take, for instance, Juan Sánchez Cotán's Quince, Cabbage, Melon and Cucumber, where the vegetables are presented in the way that accentuates the geometrical relations between them. This was meant to stimulate religious contemplation (see Bryson 1990) and treating the items as something to be eaten is not a fitting response to that painting. 
way. Thus, insofar as both still lifes and FP can make such a response possible, it is unclear why we should exclude FP images from at least potentially being artistic achievements in virtue of enabling CGI.

One could argue that FP images cannot be artistic achievements because, unlike still lifes, the pleasurable experience that one has when constructing a gustatory object in response to FP is not aesthetic. This objection assumes an aesthetic conception of artistic achievement which may be problematic anyway. But even if we grant this assumption, it doesn't seem to be true that FP cannot afford aesthetic experience. Here, I will just consider a few ways of understanding aesthetic experience and argue that CGI in response to FP can count as such under all of them.

First, take Robert Stecker's minimal characterization of aesthetic experience: "the experience of attending in a discriminating manner to forms, qualities or meaningful features of things, attending to these for their own sake or for the sake of this very experience" (Stecker 2006, p. 4). What makes an experience aesthetic under this conception is that the objects of experience or the experience itself are attended to non-instrumentally, with an expectation to find intrinsic value in them. If we take this definition on board, we can argue that an aesthetic experience of FP is possible and can even occur in virtue of the fact that FP motivated CGI. After all, those who view $\mathrm{FP}$ and engage in constructive gustatory imagination apply their ability of visual and gustatory discrimination to attend to the features of the image and to their experience of imagining the gustatory object that this image makes possible for the sake of this very experience. They do not attend to their experience for the sake of some further benefit that it might deliver. For the most part, they do not expect the imaginings of gustatory objects to help them achieve or experience something over and above those gustatory objects themselves. Attending to CSI need not involve any promise of actually eating something or of improving one's cooking skills, for instance. FP is therefore a fitting medium for aesthetic experience in this minimal sense.

An alternative understanding of aesthetic experience is in terms of its distinctive content. Noël Carroll who defends the content-based view has argued that aesthetic experience involves attending to the formal and/or expressive properties of the object and/or relations between those properties and one's responses (Carroll 2002, 2015). I am not sure what to say about the expressive properties of FP images (if they have any), but FP does seem to have the relevant 
formal properties to afford aesthetic experience in Carroll's sense. Given our characterization of constructive imaginings, these require attending to the configurational aspects of the image in order to construct the imagined gustatory object. The visual properties that are evocative of different tastes contribute to the construction of the imagined gustatory object. Therefore, since configurational aspects of the image and their relation to the gustatory features of imagined food are its formal properties, the pleasurable constructive gustatory imagination qualifies as an aesthetic experience also when one prefers the content-based view.

Another, historically prominent way of understanding aesthetic experience is that it is cut off from mundane concerns and projects. This understanding is reflected, for instance, in Kant's claim that aesthetic pleasure is disinterested: it involves attending to the form of the object without considering how the object relates to the satisfaction of one's needs and desires. ${ }^{23}$ A similar idea has been expressed in terms of distancing. As with disinterestedness, distancing means detaching oneself from the concerns that one would otherwise have (Bullough 1957). Again, the pleasure of constructive gustatory imagination seems to qualify as aesthetic because the appreciation of FP in the form of constructive imaginings does not turn on the hunger of the viewer. Such imaginings, although about food, are therefore in an important sense insulated from one's actual concerns regarding what to eat and how to satisfy one's hunger.

We don't need to decide here which conception of aesthetic experience is correct. What is important for present purposes is that under each account, pleasurable gustatory imaginings in response to FP qualify as an aesthetic experience. And if that is the case, then FP cannot be distinguished from artistic genres like still lifes in terms of not affording aesthetic experiences.

One could retort that, unlike FP, still lifes are created with an artistic purpose in mind and thereby can be artistic achievements when that purpose is realized. The tricky issue here is to understand what having an artistic purpose exactly amounts to. If we define it very narrowly in terms of an intention to present something to the artworld, for instance, where the artworld is a community of people who engage in art creation and criticism and have a particular

\footnotetext{
${ }^{23}$ Kant's own understanding of disinterestedness seems to be that in aesthetic engagement, the agent focuses on the appearance of the object, without being concerned with its existence (Sweeney 2012, p. 54). A focus on the appearance and disregard for the reality is also noticeable in the case of CGI that FP affords.
} 
history, then it may be possible to sever the analogy between FP and art kinds. There is arguably no settled historical tradition of FP. But is it really plausible that one cannot try to achieve artistic excellence independently of an intention to present something to the artworld public? At least some creators of FP do seem to aim at technical and skillful presentation, using photographic medium and preproduction manipulation to convey the enticing aspects of food. I do not see any good reasons to preclude this from counting as an artistic purpose. It is reasonable to assume that at least some of them intend their images to be enjoyed not merely for the sake of arousing or enhancing hunger but to be enjoyed for their own sake. And one form which enjoying for its own sake takes is constructive gustatory imagination. FP is a fitting vehicle for this.

\section{Comparative Artistic Merit of FP}

FP and still lifes can therefore be similar with respect to their contents, with respect to the experience that they afford and with respect to the artistic involvement. If we grant this, can different FP images be comparatively evaluated in terms of artistic merit? I am not sure if there is a clear recipe for this, but we can distinguish between levels of sophistication in gustatory imaginings that different FP images afford. Arguably, there is a correlation between the artistic value of an artwork and the complexity of the response it affords. Even in the case of primitivist or naïvist artworks of high artistic merit, one has to have sufficient knowledge of art history and a good sense of discrimination to fully appreciate them. In the remaining part of the section, I will argue that some FP images call for more sophisticated responses than others and this makes them artistically more valuable.

Mohan Matthen (2015) has drawn a useful distinction between primary and secondary sensory attractors of artworks. Primary attractors are features which appeal to those who perceive them without the latter having to learn to appreciate them. That people find those attractors appealing is presumably a human universal. Matthen's examples of primary attractors involve melodic harmonies, certain visual patterns and colors and graceful movement (Matthen 2015, p. 174). Secondary attractors, on the other hand, are particular ways in which primary attractors are used and require learning in order to be appreciated. They are reflected in an artwork's style and form, and they imply standards of evaluation which a naïve viewer fails to grasp. Secondary attractors can also outweigh primary attractors: in acquiring a more refined taste, what one used to consider beautiful 
in terms of primary attractors may begin to appear lame, shallow and schmaltzy in terms of secondary attractors. Artworks that appeal to secondary attractors over primary ones can therefore be taken to generally have higher artistic merit than those that don't or that only have primary attractors. ${ }^{24}$

The distinction between primary and secondary attractors can also be applied to the perception of food. Primary sensory attractors of food arguably involve those properties that suggest high energetic value. After all, in the environment in which our brains evolved, it was crucial to find food which would sustain one's energy levels. Since sweetness indicates high energetic value, people find sweet tastes appealing without any perceptual learning. Bitterness, on the other hand, is not a primary attractor, given that it is indicative of toxins (Holmes 2017, p. 16). The visual properties of foods we see are naturally associated with particular flavors and can thus also function as gustatory attractors, at least in a derived sense. The primarysecondary distinction also applies to derived gustatory attractors, in that we don't find those visual properties that suggest bitterness naturally appealing. However, visual properties that are associated with flavors that are not naturally appealing can be employed in secondary attractors. For instance, someone who has learned to appreciate bitter ales and spicy foods can appreciate also visual properties that suggest some kinds of bitterness and spiciness.

If we now consider food imagery, there is evidence which suggests that our brains are hardwired to track the energetic value of not only foods that we see but also foods that we see in images (Toepel et al. 2009). This explains the natural attraction of FP images that depict sweet and fatty foods (Spence et al. 2016). And again, the distinction between primary and secondary attractors can be applied to food images. Primary attractors in food images are those visual properties that we associate with primary gustatory attractors. The constructive imaginings that are prompted by those attractors also result in naturally attractive gustatory objects. Arguably, most FP images appeal to primary attractors which is why images of sweet and fatty foods dominate Instagram and Pinterest. But the dominance of primary attractors in FP does not preclude the possibility of FP images that accentuate secondary attractors, in which case the constructive imaginings require learning and sophistication from their viewers. It is

\footnotetext{
${ }^{24}$ This is not to say that an artwork which accentuates primary attractors is always of low artistic merit, it might be highly valuable, all things considered. That secondary attractors overweigh primary ones is a pro tanto claim. I thank an anonymous reviewer for pressing me on this.
} 
possible to develop skills in FP appreciation through learning. By associating new gustatory properties with particular visual configurations and learning to enjoy foods that one didn't appreciate before, one can learn to treat new kinds of FP images as a source of constructive gustatory imagination. While such images are not such that they could invoke an imaginative response in a naïve audience, they do induce it in those who have gone through the relevant learning process. Although affording a sophisticated response, these images still function to arouse the viewer and therefore fall under the definition of FP that we gave in the beginning of this paper.

Since secondary attractors have a priority over primary ones in determining the artistic value of an object, we can then say that FP images can be comparatively evaluated in terms of the extent to which they involve secondary attractors and prompt sophisticated gustatory imaginings. We might then perhaps even say that a person who prioritizes primary gustatory attractors in their FP appreciation has a bad taste in FP.

\section{Conclusion}

In this paper, I have argued that the pleasures that one can take in FP are diverse and do not just consist in blind arousal. In fact, what FP is in a particularly good position to afford are pleasurable imaginings that construct a gustatory object in response to what is seen in FP images. FP makes thereby possible cross-modal gustatory experiences which it might not be possible to have with the food that was the causal source of the image. This makes it more understandable why so many people are attracted to those images: they afford something like a (virtual) gustatory pleasure, not merely an enjoyment of the visual. What is more, FP images can also be evaluated in terms of their artistic merit, depending on the inclusion of secondary attractors and the sophistication of the gustatory imaginings that an image induces.

The claims about the artistic potential of FP have been somewhat promissory, though, in that they have been about the possibilities of FP, not about actual examples. It might be the case that FP that has been created thus far has little artistic value. But there is nothing in the medium of FP that precludes it from having artistic worth. In particular, the use of digital photography by which one can manipulate and enhance FP images provides countless possibilities of expanding the range of gustatory objects that we can imagine. ${ }^{25}$

${ }^{25}$ I am grateful to the audience at the Conference on Food, Art, and Philos- 


\section{REFERENCES}

Barrett, D., 2010, Supernormal Stimuli: How Primal Urges Overran Their Evolutionary Purpose, W.W. Norton and Company, New York/London.

Briscoe, R., 2018, "Superimposed Mental Imagery: On The Uses of MakePerceive", in F. Dorsch and F. Macpherson (eds.), Perceptual Memory and Perceptual Imagination, Oxford University Press, Oxford, pp. 161185.

Bryson, N., 1990, Looking at the Overlooked: Four Essays on Still Life Painting, Harvard University Press, Cambridge, MA.

Bullough, E., 1957, Aesthetics: Lectures and Essays, Bowes and Bowes, London.

Carroll, N., 2015, "Defending the Content Approach to Aesthetic Experience", Metaphilosophy, vol. 46, no. 2, pp. 171-188.

- 2002, "Aesthetic Experience Revisited", The British Journal of Aesthetics, vol. 42, no. 2, pp. 145-168.

Coward, R., 1984, Female Desires: How They Are Sought, Bought and Packaged, Grove Weidenfeld, New York.

Currie, G., 2010, “Tragedy”, Analysis, vol.70, no. 4, pp. 632-638.

Doggett, T. and A. Egan, 2012, "How We Feel about Terrible, Non-Existent Mafiosi", Philosophy and Phenomenological Research, vol. 84, no. 2, pp. 277-306.

Elder R.S. and A. Krishna, 2012, "The Visual Depiction Effect in Advertising: Facilitating Embodied Mental Simulation through Product Orientation", Journal of Consumer Research, vol. 38, pp. 988-1003.

Heathwood, C., 2007, "The Reduction of Sensory Pleasure to Desire", Philosophical Studies, vol. 133, no. 1, pp. 23-44.

Holmes, B., 2017, Flavor: The Science of Our Most Neglected Sense, W.W. Norton and Company, New York/London.

Kim, Y., 2018, "Sell your Loneliness: Mukbang Culture and Multisensorial Capitalism in South Korea", in L. Lim and H.-K. Lee (eds.), Routledge Handbook of Cultural and Creative Industries in Asia, Routledge, pp. 225-238.

Korsmeyer, C., 2002, Making Sense of Taste: Food and Philosophy, Cornell University Press, Ithaca/London.

Kozinets, R., A. Patterson and R. Ashman, 2016, "Networks of Desire: How Technology Increases our Passion to Consume", Journal of Consumer Research, vol. 43, no. 5, pp. 659-682.

Levinson, J., 2005, "Erotic Art and Pornographic Pictures", Philosophy and Literature, vol. 29, no. 1, pp. 228-240.

ophy at National Autonomous University of Mexico for helpful comments and questions. Special thanks to Paloma Atencia, Ricardo Mena, Shen-yi Liao, Aaron Meskin, Kengo Miyazono and two anonymous referees. The research in this paper was supported by JSPS Postdoctoral Fellowship for Research in Japan (Standard), KAKENHI (19F19762). 
McDonnell, E.M., 2016, "Food Porn: The Conspicuous Consumption of Food in the Age of Digital Reproduction", in P. Bradley (ed.), Food, Media and Contemporary Culture: The Edible Image, Palgrave Macmillan, London, pp. 239-265.

Maes, H., 2011, “Art or Porn: Clear Division or False Dilemma?", Philosophy and Literature, vol. 35, no. 1, pp. 51-64.

Matthen, M., 2015, "Play, Skill, and The Origins of Perceptual Art", British Journal of Aesthetics, vol. 55, no. 2, pp. 173-197.

Nguyen, C.T. and B. Williams, 2020, "Moral Outrage Porn", Journal of Ethics and Social Philosophy, vol. 18, no. 2, pp. 147-172.

Petit, O., A.D. Cheok and O. Oullier, 2016, "Can Food Porn Make Us Slim? How Brains of Consumers React to Food in Digital Environments", Integrative Food, Nutrition and Metabolism, vol. 3, pp. 251-255.

Piech, R.M., M.T. Pastorino and D.H. Zald, 2010, "All I Saw Was the Cake. Hunger Effects on Attentional Capture by Visual Food Cues", Appetite, vol. 54, pp. 579-582.

Piqueras-Fiszman, B. and C. Spence, 2012, "The Influence of the Color of the Cup on Consumers' Perception of a Hot Beverage", Journal of Sensory Studies, vol. 27, no. 5, pp. 324-331.

Podro, M., 1998, Depiction, Yale University Press, London.

Prescott, J., 2015, "Multisensory Processes in Flavour Perception and Their Influence on Food Choice", Current Opinion in Food Science, vol. 3, pp. $47-52$.

Prince, R., 2014, "How We're Fed 434 Hours of TV Cookery a Week But the More They Show, the Less We Cook", Daily Mail Online, 26th September. Downloaded from <https://www.dailymail.co.uk/tvshowbiz/ article-2771553/How-fed-434-hours-TV-cookery-week-cook.html $>$ [accessed: $01 / 11 / 2018]$

Rea, M.C., 2001, "What Is Pornography?", Noûs, vol. 35, no. 1, pp. 118145.

Rousseau, S., 2014, "Food "Porn" in Media", in P.B. Thompson and D.M. Kaplan (eds.), Encyclopedia of Food and Agricultural Ethics, Springer Netherlands, pp. 748-754.

Schopenhauer, A., 1969 [1819], The World as Will and Representation, vol. 1, trans. E.E. Payne, Dover, New York.

Simmons, W.K., A. Martin and L.W. Barsalou, 2005, "Pictures of Appetizing Foods Activate Gustatory Cortices for Taste and Reward", Cerebral Cortex, vol. 15, no. 10, pp. 1602-1608.

Sinhababu, N., 2017, Humean Nature: How Desire Explains Action, Thought, and Feeling, Oxford University Press, New York.

Small, D.M. and J. Prescott, 2005, "Odor/Taste Integration And the Perception of Flavor, Experimental Brain Research", vol. 166, no. 34, pp. 345-357.

Spence, C., 2017, Gastrophysics: The New Science of Eating, Viking, New York. 
Spence, C., 2015, "On the Psychological Impact of Food Colour”, Flavour, vol. 4, no. 21, pp. 1-6.

Spence, C., K. Okajima, A.D. Cheok, O. Petit and C. Michel, 2016, "Eating with Our Eyes: From Visual Hunger to Digital Satiation", Brain and Cognition, vol. 110, pp. 53-63.

Spence, C. and B. Piqueras-Fiszman, 2014, The Perfect Meal: The Multisensory Science of Food and Dining, John Wiley and Sons, New Jersey.

Spence, C., C.A. Levitan, M.U. Shankar and M. Zampini, 2010, "Does Food Color Influence Taste and Flavor Perception in Humans?", Chemosensory Perception, vol. 3, no. 1, pp. 68-84.

Stecker, R. 2006, "Aesthetic Experience and Aesthetic Value", Philosophy Compass, vol. 1, no. 1, pp. 1-10.

Sweeney, K.W., 2012, "Hunger Is the Best Sauce: the Aesthetics of Food", in D.M. Kaplan (ed.), The Philosophy of Food, University of California Press, Berkeley, pp. 52-68.

Toepel, U., J.F. Knebel, J. Hudry, J. le Coutre and M.M. Murray, 2009, "The Brain Tracks the Energetic Value in Food Images", Neuroimage, vol. 44, no. 3, pp. 967-974.

Uidhir, C.M., 2009, "Why Pornography Can't Be Art", Philosophy and Literature, vol. 33, no. 1, pp. 193-203.

Van Brabandt, P. and J. Prinz, 2012, "Why do Porn Films Suck?", in H. Maes and J. Levinson (eds.), Art and Pornography: Philosophical Essays, Oxford University Press, Oxford, pp. 161-190.

Velasco, C., M. Obrist, O. Petit and C. Spence, 2018, "Multisensory Technology for Flavor Augmentation: A Mini Review", Frontiers in Psychology, vol. 9, pp. 1-6.

Received: December 20, 2019; revised: April 27, 2020; accepted: July 11, 2020. 